\title{
AVALIAÇÃO DO USO DE ALBUMINA HUMANA EM UM HOSPITAL UNIVERSITÁRIO DO NORDESTE BRASILEIRO
}

\author{
F. P. B. F. CARDOSO ${ }^{1}$, H. L. SILVA-NETA ${ }^{2}$, M. M. VIANA ${ }^{1}$, R. A. D. M. SOUTO ${ }^{1}$, J. P. \\ A. CARDOSO 3 , R. A. MELO ${ }^{4}$, A. C. de MEDEIROS \\ ${ }^{1}$ Universidade Federal da Paraíba, Hospital Universitário Lauro Wanderley, Unidade de \\ Farmácia Clínica \\ ${ }^{2}$ Universidade Federal de Pernambuco, Núcleo de Pesquisa em Inovação Terapêutica Suely \\ Galdino, Laboratório de Imunomodulação e novas abordagens terapêuticas \\ ${ }^{3}$ Instituto Federal da Paraíba \\ ${ }^{4}$ Universidade Estadual da Paraíba \\ ${ }^{5}$ Universidade Federal da Paraíba, Centro de Ciências Médicas, Departamento de Medicina \\ Interna
}

E-mail para contato: flavinhafarm@hotmail.com

\begin{abstract}
RESUMO - O objetivo deste estudo foi verificar a conformidade das prescrições de albumina humana tendo em vista as diretrizes da Agência Nacional de Vigilância Sanitária (ANVISA), em um Hospital Universitário de nível terciário. Para tanto, foi realizada a análise das prescrições encaminhadas a farmácia hospitalar no período de julho a dezembro de 2011. As indicações constantes nas prescrições foram classificadas de acordo com a RDC $n^{\circ} 115$ em formais, discutiveis e não fundamentadas. Foi encontrada a seguinte distribuição de indicações: 42,8\% formais, 8,6\% discutíveis, 40\% não fundamentadas e 8,6\% de indicações não contempladas pela RDC. Ao realizar a análise da quantidade de frascos de albumina humana dispensados por grupo de indicação, foi verificado que o maior percentual (51\%), foi dispensado para uso em indicações não fundamentadas. Em síntese, apesar de a maioria das indicações de albumina estarem de acordo com o grupo das indicações formais da $R D C n^{\circ} 115$, o maior percentual de frascos utilizados foi destinado ao uso não fundamentado deste medicamento. Nossos dados demonstram a necessidade de construção de um protocolo clínico institucional para uso da albumina humana, com a participação da equipe multiprofissional, que contemple além das indicações, a dose, o tempo de tratamento e os objetivos do mesmo a fim de evitar o seu uso não fundamentado e consequentes gastos desnecessários.
\end{abstract}

Palavras-chave: Terapia Medicamentosa. Hemoderivados. Protocolo clínico.

ABSTRACT - The objective of this study was to verify compliance of human albumin prescriptions in accordance with National Agency for Sanitary Surveillance (ANVISA) guidelines at a tertiary-level university hospital. To do so, the analysis of the prescriptions sent to the hospital pharmacy was carried out from July to December 2011. The indications included in the prescriptions were classified according to RDC number 115 in formal, debatable and unsubstantiated. The following distribution of indications 
was found: $42.8 \%$ formal, $8.6 \%$ debatable, $40 \%$ unsupported and $8.6 \%$ of indications not contemplated by the RDC. When analyzing the amount of human albumin vials dispensed per indication group, it was verified that the highest percentage (51\%) was dispensed for use in non-substantiated indications. In summary, although the majority of albumin indications are in agreement with the group of formal indications of RDC number 115 , the highest percentage of vials used were intended for the unsubstantiated use of this drug. Our data demonstrate the need to construct a clinical institutional protocol for the use of human albumin, with the participation of the multiprofessional team, which includes besides the indications, the dose, the time of treatment and the objectives of the same in order to avoid its use not substantiated and consequent unnecessary expenses.

Key words: Drug Therapy. Blood-Derivative Drugs. Clinical protocol.

\section{INTRODUÇÃO}

A albumina é uma proteína natural presente no plasma sanguíneo. Por ser de fácil purificação, ela foi uma das primeiras a serem estudadas pelos cientistas, o que favoreceu seu uso clínico desde a década de 1940 nos Estados Unidos (PDB, 2012; MAGALHÃES e CHINI, 2007, RAZOUK e REICHE, 2004). Esta proteína é uma molécula relativamente pequena, sintetizada pelo fígado, de ampla distribuição, que tem sua função principal associada a manutenção da pressão oncótica intravascular. Além desta função ela também está associada ao transporte de substâncias vitais e a inativação de substâncias tóxicas (BORGES FILHO, et al, 2010).

A albumina existe no mercado farmacêutico compondo o arsenal dos medicamentos derivados do sangue, os hemoderivados. Sob esta forma, a albumina humana é definida como "uma solução proteica, estéril, e apirogênica, obtida por fracionamento de plasma e que corresponde eletroforeticamente à fração albumina do plasma humano" (BRASIL, 2000). Este hemoderivado é produzido a partir de grandes quantidades de plasma submetidas a fracionamento a frio pelo etanol. O produto inicial é submetido a um demorado processo de esterilização pelo calor para a eliminação de vírus e bactérias. Este rigoroso cuidado no processamento da albumina contribui para o excelente grau de segurança do seu uso clínico (BRASIL, 2008).

$\mathrm{Na}$ clínica, a albumina é empregada como medicamento essencial, e seu uso objetiva a expansão de volume intravascular e a manutenção do débito cardíaco (BIOLO, 2004). Por ser um produto de difícil obtenção e, portanto, de alto custo, faz-se necessário o uso racional da albumina humana, seguindo para tanto diretrizes oficiais que regulamentem o seu uso. No Brasil, a Resolução de Diretoria Colegiada (RDC) $n^{0} 115$, de 10 de maio de 2004, estabelece as diretrizes para o uso da albumina humana (BRASIL, 2004). No entanto, acredita-se que a adesão, por parte dos profissionais prescritores, a tais diretrizes é baixa. Portanto, o presente estudo visa verificar a conformidade das prescrições de albumina humana tendo em vista as diretrizes da Agência Nacional de Vigilância Sanitária (ANVISA) em um Hospital Universitário de nível terciário.

\section{MATERIAIS E MÉTODOS}

Trata-se de um estudo transversal, retrospectivo e quantitativo que utilizou como fonte de informações os laudos de procedimentos especiais preenchidos para solicitação 
de albumina humana a farmácia de um Hospital Universitário. No local do estudo a albumina humana está disponível na forma de solução de albumina a $20 \%$ em frascos de $50 \mathrm{~mL}$ e todos os pedidos são, obrigatoriamente, solicitados por meio do preenchimento do referido laudo, o qual passaremos a chamar de prescrição neste estudo. Foram incluídos no estudo todos as prescrições aviadas no período de julho a dezembro de 2011. Prescrições ilegíveis, com preenchimento incorreto e com indicação não definida foram excluídas.

Para análise das indicações do uso de albumina humana utilizou-se como base a RDC $\mathrm{n}^{\circ} 115$ de 10 de maio de 2004, que aprova as diretrizes para o uso de albumina. As indicações para as quais a albumina foi utilizada no hospital foram classificadas de acordo com a RDC $\mathrm{n}^{\circ} 115$ em formais, discutíveis e não fundamentadas. As indicações não contempladas no protocolo supracitado foram classificadas como "não se enquadra".

Os dados foram tabulados no software Microsoft Excel ${ }^{\circledR}$ para Windows versão 2007 e analisados estatisticamente através do programa SPSS (Statistical Package for Social Sciences) for Windows versão 18.0. A pesquisa foi aprovada pelo Comitê de Ética em Pesquisa do Hospital Universitário Lauro Wanderley de acordo com o protocolo $\mathrm{n}^{\circ} 510 / 11$.

\section{RESULTADOS E DISCUSSÃO}

Durante o período do estudo, foram enviadas a farmácia 71 prescrições de albumina humana, sendo 01 excluída da amostra por não apresentar a justificativa de uso do medicamento. Portanto, o estudo contou com 70 prescrições.

Foi verificado que o maior percentual de indicações foi formal $(42,8 \%)$, como mostra a tabela 1. No entanto é importante ressaltar que $40 \%$ das indicações foram classificadas como não fundamentadas. Tal resultado deve ser levado em consideração tendo em vista o alto custo associado a este medicamento.

Tabela 1 - Classificação das prescrições de Albumina Humana de acordo com a RDC n ${ }^{\circ}$ 115

\begin{tabular}{lcc}
\hline \multicolumn{1}{c}{ Classificação } & Frequência $(\mathbf{n}=\mathbf{7 0})$ & Percentual (\%) \\
\hline Formal & 30 & 42,8 \\
Discutível & 6 & 8,6 \\
Não fundamentada & 28 & 40,0 \\
Não se enquadra* & 6 & 8,6 \\
\hline Total & $\mathbf{7 0}$ & $\mathbf{1 0 0}$ \\
\hline
\end{tabular}

*prescrições inadequadas que não se enquadram na classificação da RCD n 115.

Foi verificado ainda, um baixo percentual de indicações que não se enquadraram na RDC $\mathrm{n}^{\circ} 115$, contrapondo-se aos resultados encontrados em estudo semelhante, conduzido por Magalhães e Chini (2007), onde os autores concluíram que a grande maioria dos pedidos para a reposição de albumina não tem embasamento científico e não consta das indicações da Resolução ${ }^{\circ} 115$ da ANVISA. Apesar de tais usos não estarem contemplados na resolução, acredita-se que estejam baseados em experiências clínicas.

Estudo publicado por Matos e Rozenfeld (2005), que avaliou o uso de albumina humana em um Hospital Geral Federal do Rio de Janeiro, utilizando uma síntese de protocolos, elaborada com base em quatro protocolos padrão de países diferentes incluindo a $\operatorname{RDC} \mathrm{n}^{\circ} 115$, encontrou um percentual maior de indicações inapropriadas 
$61,8 \%$, seguido de $33,1 \%$ apropriadas e 4,6\% controversas. Opondo-se aos resultados do presente estudo, que encontrou um maior percentual de indicações apropriadas.

Os achados referentes às indicações não fundamentadas corroboram com a análise prospectiva preliminar realizada no estudo de Borges Filho et al. (2010) que encontrou um percentual de $42 \%$ das prescrições classificadas como não fundamentadas, ou seja, aquelas em que os trabalhos mostram que o uso da albumina não traz nenhum benefício para os pacientes.

Foi realizada a análise percentual das indicações dentro de cada grupo. No grupo das indicações formais, que correspondeu a 30 prescrições, 46,7\% das mesmas foram direcionadas para o tratamento de pacientes com ascites volumosas, por paracenteses repetidas; 43,3\% para uso após paracenteses evacuadoras nos pacientes com ascites volumosas e $10 \%$ para pacientes com cirrose hepática e síndrome nefrótica, quando houveram edemas refratários aos diuréticos e com risco iminente a vida dos mesmos.

No grupo das indicações discutíveis, todas as prescrições $(n=6)$ foram direcionadas a pacientes críticos com hipovolemia, hipoalbuminemia e má-distribuição hídrica.

Já no grupo das indicações não fundamentadas, que contou com 28 prescrições, $53,6 \%$ das mesmas foram direcionadas para o tratamento de pacientes com cirrose hepática ou com síndrome nefrótica; 39,3\% para correção de hipoalbuminemia e 7,1\% para peri-operatório de outros casos não contemplados pela $\mathrm{RDC} \mathrm{n}^{\circ} 115$. Os resultados descritos são mostrados na tabela 2 .

Tabela 2 - Percentual do uso de albumina humana dentro de cada grupo das indicações presentes na RDC $n^{\circ} 115$.

\begin{tabular}{|c|c|c|}
\hline Classificação & $\begin{array}{c}\text { Frequência } \\
(n=64)\end{array}$ & $\begin{array}{l}\text { Percentual } \\
\quad(\%)\end{array}$ \\
\hline \multicolumn{3}{|l|}{ Formal $(n=30)$} \\
\hline $\begin{array}{l}\text { Tratamento de pacientes com ascites volumosas, } \\
\text { por paracenteses repetidas. }\end{array}$ & 14 & 46,7 \\
\hline $\begin{array}{l}\text { Após paracenteses evacuadoras nos pacientes com } \\
\text { ascites volumosas. }\end{array}$ & 13 & 43,3 \\
\hline $\begin{array}{l}\text { Pacientes com cirrose hepática e síndrome } \\
\text { nefrótica, quando houver edemas refratários aos } \\
\text { diuréticos e que coloquem em risco iminente a } \\
\text { vida dos pacientes. }\end{array}$ & 3 & 10 \\
\hline \multicolumn{3}{|l|}{ Discutível $(n=6)$} \\
\hline $\begin{array}{l}\text { Em pacientes críticos com hipovolemia, } \\
\text { hipoalbuminemia e má-distribuição hídrica. } \\
\text { Não Fundamentada }(\mathbf{n}=\mathbf{2 8})\end{array}$ & 6 & 100 \\
\hline Correção de hipoalbuminemia. & 11 & 39,3 \\
\hline $\begin{array}{l}\text { Tratamento de pacientes com cirrose hepática ou } \\
\text { com síndrome nefrótica. }\end{array}$ & 15 & 53,6 \\
\hline $\begin{array}{l}\text { Peri-operatório, exceto nos casos mencionados } \\
\text { anteriormente. }\end{array}$ & 2 & 7,1 \\
\hline Total & 64 & 100 \\
\hline
\end{tabular}

Em estudo conduzido por Borges et al. (2010), dentre as indicações formais as principais ocorrências foram o uso após paracenteses evacuadoras nos pacientes com ascites volumosas e em pacientes com cirrose hepática e síndrome nefrótica, quando 
houver edemas refratários aos diuréticos e que coloquem em risco iminente a vida dos pacientes. Diferente do nosso estudo, em que o maior percentual foi para tratamento de pacientes com ascites volumosas, por paracenteses repetidas.

Quanto à classificação discutível, em sua totalidade (100\%) foi observado o uso em pacientes críticos com hipovolemia, hipoalbuminemia e má-distribuição hídrica. De acordo com Lee (2012), o uso para este fim tornou-se discutível após a publicação da revisão de Cochrane que concluiu que a administração de albumina em pacientes gravemente enfermos pode aumentar o risco de morte. A autora relata ainda que nenhuma diferença significativa quanto a morbidade, tempo de permanência na unidade de cuidados intensivos ou hospital e aumento de sobrevida foi evidenciado quando se administrou albumina ao invés de solução fisiológica como fluido de reanimação.

Dentre as indicações não fundamentadas, a administração de albumina para o tratamento de pacientes com cirrose hepática ou com síndrome nefrótica obteve o maior percentual (53,6\%). De acordo com Boldt (2010), na síndrome nefrótica a albumina é perdida através dos rins e a compensação da hipoalbuminemia resultante através da administração do medicamento hemoderivado não é útil, uma vez que o mesmo também será eliminado pelos rins. O mesmo autor relata, ainda, que não existem evidências de que o uso da albumina traga benefícios no tratamento de pacientes com cirrose hepática.

A análise quantitativa dos dados mostrou que foram dispensados 575 frascos de albumina humana. A análise estatística evidenciou uma média de $8,2 \pm 8,8$ frascos de albumina por prescrição, com valor mínimo de 01 frasco e máximo de 60 frascos dispensados.

Sendo o valor mínimo de 01 frasco utilizado para quadro de hipoalbuminemia em pacientes pediátricos e desnutrição severa em paciente adulto e o valor máximo de 60 frascos utilizados para desnutrição grave em paciente adulto. De acordo com a literatura, a albumina humana não é uma boa fonte proteica, principalmente quando comparada às soluções parenterais de aminoácidos e aos lisados proteicos das soluções enterais, e não há elementos que justifiquem a utilização da albumina para correção de hipoalbuminemia

(BORGE FILHO et al., 2010).

Tendo por base a distribuição percentual da quantidade de frascos de albumina humana dispensados levando em consideração o grupo de indicação ao qual pertencia, verificamos que o grupo das indicações não fundamentadas consumiu um maior número de frascos de albumina humana (51\%), seguido da indicação fundamentada (36\%), das prescrições que não se enquadraram na RDC n $115(7,4 \%)$ e das indicações discutíveis $(5,6 \%)$. Estes resultados podem ser verificados na tabela 3 .

Tabela 3 - Quantidade de frascos dispensados por classificação de indicação

\begin{tabular}{lcc}
\hline \multicolumn{1}{c}{ Classificação } & Frasco-ampola (n=575) & Percentual (\%) \\
\hline Formal & 206 & 36,0 \\
Discutível & 32 & 5,6 \\
Não fundamentada & 294 & 51,0 \\
Não se enquadra & 43 & 7,4 \\
\hline Total & $\mathbf{5 7 5}$ & $\mathbf{1 0 0}$ \\
\hline
\end{tabular}


Apesar de termos verificado um maior número de prescrições formais, fazendo a análise a partir da quantidade de frascos de albumina dispensados por grupo de indicações, verificamos que o uso não fundamentado apresentou um maior consumo.

A análise dos gastos associados a este medicamento não constituiu objetivo deste estudo, no entanto, através dos resultados descritos, pode-se observar que os gastos com uso não fundamentado de albumina são maiores que os gastos com o uso fundamentado. Estima-se um gasto de $\mathrm{R} \$ 16.052,40$ com o uso do medicamento para indicações não fundamentadas, durante o período deste estudo. É importante notar que uma grande economia poderia ser feita através da instituição de protocolo para uso de albumina humana e do acompanhamento multiprofissional das prescrições deste medicamento, como foi demostrado em estudo de intervenção realizado por Borges Filho et al. (2010), no Hospital Israelita Albert Einstein, no qual impacto econômico em relação ao uso desnecessário de Albumina representou economia de R \$ 825.000.

\section{CONCLUSÃO}

A partir do estudo realizado verificamos que apesar de a maioria das indicações de albumina estarem de acordo com o grupo das indicações formais da $\mathrm{RDC}^{\circ} 115$, o maior percentual de frasco utilizados $(51 \%)$ foram destinados ao uso não fundamentado deste medicamento, o que acarretou gastos desnecessários estimados em $\mathrm{R} \$ 16.052,40$.

Sugerimos a construção de protocolo clínico institucional para uso da albumina humana, com a participação da equipe multiprofissional, que contemple além das indicações, a dose, o tempo de tratamento e os objetivos do mesmo a fim de evitar o seu uso não fundamentado e consequentes gastos desnecessários para o hospital.

\section{REFERÊNCIAS}

BIOLO, A. Avaliação de Tecnologias em Saúde: sumário das Evidências e Recomendações para o Uso da Albumina Humana. Porto Alegre, 2004. Disponível em: $<$ http://www.unimedvtrp.com.br/autoriza/evidencias_aprovadas/recomendacoes_albumi na.pdf> Acesso: 22 nov. 2011.

BOLDT, J. Use of albumin: an update. Br. J. Anaesth., v.104, n.3, p. 276-84, 2010.

BORGES FILHO, W. N.; ALMEIDA, S. M.; FERRACINI, F. T.; FERNANDES JÚNIOR, C. J. Contribuição da farmácia na prescrição e uso racional de albumina humana em um hospital de grande porte. Einstein (São Paulo), v. 8, n. 2, p. 215-220, 2010.

BRASIL. Ministério da Saúde. Agência Nacional de Vigilância Sanitária. Resolução RDC n ${ }^{\circ}$ 46, de 18 de maio de 2000. Regulamenta a Produção e Controle de Qualidade dos Produtos Hemoderivados de Uso Humano, Brasília, DF, 11 maio 2004. Disponível em:

$<$ http://bvsms.saude.gov.br/bvs/saudelegis/anvisa/2000/anexo/anexo_res0046_18_05_2 000.pdf>. Acesso em: 30 nov. 2017.

BRASIL. Ministério da Saúde. Agência Nacional de Vigilância Sanitária. Resolução RDC $\mathrm{n}^{\circ} 115$, de 10 de maio de 2004. Aprova as Diretrizes para o uso de Albumina. Diário Ofícial da União, Brasília, DF, 11 maio 2004. Disponível em: < 
http://redsang.ial.sp.gov.br/site/docs_leis/rs/rs12.pdf>. Acesso em: 29 nov. 2017.

BRASIL. Ministério da Saúde. Secretaria de Atenção à Saúde. Departamento de Atenção Especializada. Guia para o uso de hemocomponentes. Brasília: Ministério da Saúde, 2008.

Lee, J. S. Albumin for End-Stage Liver Disease. Korean J. of Intern. Med., v. 27, n.1, p. 13-19, 2012.

MAGALHÃES F. A.; CHINI L. S. N. Indicações Duvidosas ou Infundadas Observadas no Tratamento com Albumina. Estudo em Hospital Universitário do Estado do Rio de Janeiro. Rev. Bras. Ter. Intensiva, v. 19, n.2, p. 192-196, 2007.

MATOS, G.C.; ROZENFELD, S. Avaliação do uso de albumina humana em hospital do Rio de Janeiro, Brasil. Cad. Saúde Pública, Rio de Janeiro, v. 21, n. 4, p. 1224-1233, 2005.

PDB (PROTEIN DATA BANK). Disponível em: $<$ http://www.rcsb.org/pdb/home/home.do.> Acesso em: 07 de jun de 2012.

RAZOUK, F. H.; REICHE, E. M. V. Caracterização, produção e indicação clínica dos principais hemocomponentes. Rev. Bras. Hematol. Hemoter., v. 26, n. 2, p. 126-134, 2004. 